\title{
IX.
}

\section{Ueber die Beziehungen der Nebennieren zu den Venen und dem reno̊sen Kreislauf.}

\author{
Aus dem Pathologischen Institut zu Strassburg i. E. \\ Von Dr. Paul Manasse, \\ II. Assistenten am Pathologischen Institut.
}

(Hierzu Taf. IV.)

Vor einiger Zeit konnte ich bei den byperplastischen Tumoren der Nebennieren nachweisen, dass erstens eine eigenthümliche Art von Zapfen und Knöpfchen, welche aus Nebennierenzellen bestanden, in die Venen eingedrungen, dass zweitens eine erhebliche Anzahl von Nebennierenzellen, innig mit Blut vermischt, in den Venen anzutreffen waren.

Da die Struktur der Tumoren vollständig der der normalen Organe glich, lag die Frage nahe, ob diese Einrichtungen der Venen auch normalen Nebennieren zukommen.

Daraufhin untersuchte ich normale menschliche Nebennieren und gelangte dabei zu folgenden Resultaten:

Wenn man zu diesen Untersuchungen keine dünnen atrophischen Organe, vielmehr solche von grossem Volumen, deren Marksubstanz nicht zerfallen, verwendet, so kann man leicht Beziehungen zwischen Nebennierensubstanz und Venen nachweisen, welche zu den bei den hyperplastischen Tumoren geschilderten vollkommen passen.

Zunächst traf ich nehmlich in den mikroskopischen Schnitten sehr häufig vereinzelte Nebennierenzellen, sowie solche, die in kleineren Haufen bei einander lagen, innig mit Blut vermischt, im Lumen der Venen an. Ferner liess sich oft genug feststellen, dass durch die Venenwandung Zapfen der Nebennierensubstanz in das Lumen hineinragten, welche ausschliesslich aus Nebennierenzellen bestanden. Diese Zapfen zeigten hinsichtlich ihrer Grösse die weitgehendsten Variationen: oft waren es nur kleine Knöpfe, dann wieder grössere solide Stränge, oft auch ganz 

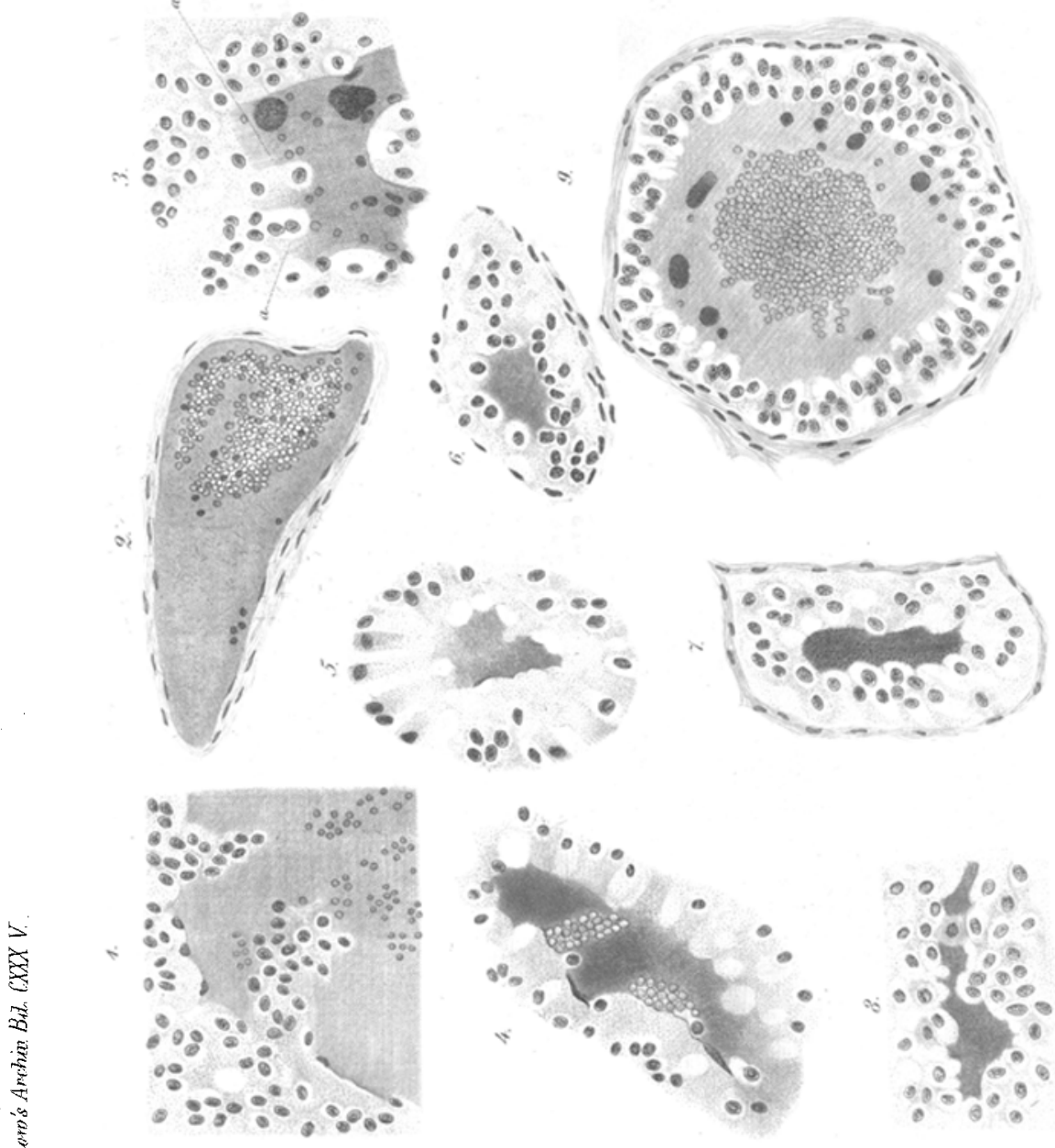
lange, scheinbar gestielte Fortsätze, welche dann jedesmal rings von Blutkörperchen umgebən waren. Gewöhnlich gingen diese Fortsätze ohne jede Grenze aus dem Parenchym der Nebennieren direct in das Venenlumen hinein, seltener waren sie von dem Parenchym durch die Venenwand getrennt, auf welcher sie dann breit aufsassen. Die grösseren dieser aus Nebennierenzellen bestehenden Zapfen, besonders diejenigen, welche mit einer kolbigen Anschwellung endeten und einen dünneren Stiel hatten, mussten wohl in dem Gefäss flottirt haben. Denn häufig hatte ich in meinen Schnitten einen solchen polypösen Fortsatz quer getroffen, so dass der Stiel fehlte, vielmehr eine Scheibe, aus Nebennierenzellen bestehend, mitten im Venenlumen, ohne die Wandung zu berühren, und vollkommen von Blnt umgeben, gelegen war.

Alle diese Dinge fanden sich gewöhnlich in der Marksubstanz, seltener in der Rinde der Nebennieren. Auch Gottschau') giebt an, dass einzelne Zellen der Marksubstanz und innersten Rindenschicht keine nachweisliche Begrenzung gegen das anliegende Gefässlumen erkennen liessen, sondern dass sie in dasselbe hineinragten; er lässt es aber unentschieden, $a b$ er solche Befunde für Kunstprodukte halten solle oder nicht. Um diese Bilder zu Gesicht zu bekommen, muss man die Schnitte nicht zu fein machen, will man nicht leere Venen antreffen; aus derselben Rücksicht sind auch keine complicirten Färbemethoden anzuwenden, überhaupt ist es das Beste mit dem Schnitt möglichst wenig zu manipuliren. Am sichersten erlangte ich jene Bilder, wenn ich die Schnitte einfach auf dem Objectträger mit Carmin oder Hämatoxylin färbte.

So konnte ich alsdann feststellen, dass eine sehr innige Beziehung zwischen der Nebennierensubstanz und den Venen besteht, der Art, dass nicht nur ein Uebertritt von Nebennierenzellen in die venöse Blutbahn stattfindet, sondern dass auch die Parenchymzapfen in das Venenlumen hineinhängen.

Nebennierenzellen, bezw. deren Zerfallsprodukte, in dem kreisenden Blute dieser Organe sind schon von Gottschau ${ }^{2}$ ) beschrieben worden. Mit Recht warnt aber Marchand ${ }^{3}$ ) davor,

1) Biolog. Centralblatt. Bd. III. S. 573.

2) a. a. O. S. 575 .

3) Internat. Beitr. z. wissensehaftl. Medic. Bd. I. S. 57 I. 
auf solche Befunde ein allzu grosses Gewicht zu legen, da nur ein geringer Druck dazu gehöre, Parenchymtheile in die Blutbahn za treiben. Da ich jedoch diese unzweifelhafte Tendenz der Nebennierenzellen, sich mit dem venösen Blute in Communication zu setzen, wenn ich so sagen darf, durch jene Zapfen anzunehmen berechtigt war, so glaubte ich mich gegenüber dem constanten Auftreten von freien Nebennierenzellen im strömenden Blute nicht allzu skeptisch verhalten zu brauchen.

Ich ging jetzt dazu über, thierische Nebennieren zu untersuchen, in der Hoffnung, ähnliche Thatsachen feststellen zu können, wie beim Menschen. Zur Untersuchung gelangten die Nebennieren von Pferden, Rindern, Kälbern, Schweinen und Schafen.

Die Ausbeute dieser Untersuchungen war viel reicher, als ich erwartet hatte. Und zwar soll uns hier in erster Linie die Marksubstanz beschäftigen.

Wenn auch die Befunde bei den einzelnen Thieren einige Verschiedenheiten zeigten, so waren doch die besprochenen Beziehungen der Venen zu dem Parenchym der Marksubstanz im Wesentlichen die gleichen. Um also Wiederholungen zu vermeiden, werde ich die Ergebnisse der Untersuchungen bei allen den genannten Thieren gemeinschaftlich besprechen und nur besondere Einzelheiten hervorheben, welche noch ein specielleres Interesse verdienen.

Was die Methodik betrifft, so sah ich mich gloich nach den ersten Untersuchungen, aus Gründen, welche weiter unten erhellen werden, genöthigt, dafür $\mathrm{zu}$ sorgen, dass die Nebennieren sofort nach dem Schlachten, im Schlachthause lebenswarm fixirt wurden. Als Fixationsmittel dienten Alkohol, Müller'sche Flüssigkeit und 2procentige Kal. bichrom.-Lösung.

Die anatomischen Verhältnisse der Marksubstanz sind oft und genau beschrieben worden; ich weise auf die Arbeiten von Eberth ${ }^{1}$ ), v. Brunn ${ }^{2}$ ) and Arnold ${ }^{3}$ ) hin.

Dieselbe setzt sich zusammen aus einem bindegewebigen Gerüste, welches sehr stark von Venen und venösen Räumen

1) In Stricker's Handbuch der Gewebelehre. S. 512 ,

2) Schulze's Archiv. Bd. 8. S. 618 .

3) Dieses Arehiv. Bd. 35. S. 64, 
durchzogen ist, auch liegen zahlreiche Nerven sowie vereinzelte Ganglienzellen darin.

Das bindegewebige Gerüst wird eingenommen von Zellcomplexen, welche je nach der Schnittrichtung verschiedene Anordnung zeigen. Gewöhnlich sind die Zellen in Haufen und Strängen angeordnet, deren Form sich der des bindegewebigen Gerüstes vollständig anpasst. Die Haufen sind theils rundlich, theils länglich, letzteres vorherrschend, liegen in keiner bestimmten Anordnung, sondern kreuzen sich mehrfach innerhalb der Marksubstanz. Diese länglichen Zellbaufen zeigen häufig folgende Struktur: Eine dünne cylindrische bindegewebige Hülle umschliesst eine Doppelreihe von Parenchymzellen, welche mit der einen Seite dem bindegewebigen Septum fest aufsitzen, mit der anderen die gegenüber liegenden Zellen berühren; häufig oder vielmehr gewöhnlich ist hier die Berührung eine so innige, dass der Inhalt der einen Zelle in den der gegenüber liegenden übergeht, ein Verhältniss, welches auch die neben einander an der bindegewebigen Wand aufgepflanzten Zellen zeigen. Bei der Membranlosigkeit der Elemente, die ja auch v. Brunn ${ }^{1}$ ) erwähnt, kann das nicht weiter Wunder nehmen. Ich möchte jedoch bemerken, dass ich nicht selten solche bindegewebigen Schläuche angetroffen habe, welche nur eine einfache Reihe von Zellen einschlossen. Dio Zellen selbst haben ein entschieden epitheliales Aussehen und sind theils von kubischer, theils von cylindrischer Form.

Charakteristisch für diese Zellen ist es, dass sie einen tiefbraunen Farbenton annehmen, wenn man die Nebennieren mit Lösungen von chromsauren Salzen behandelt.

Auf diese Thatsache haben früher v. Brunn und Andere aufmerksam gemacht; in neuester Zeit hat H. Stilling ${ }^{2}$ ) diese Reaction benutzt, um die gleichen Zellen in der Carotisdrüse nachzuwoisen. Doch möchte ich hier gleich bemerken, dass die Marksubstanzzellen nicht immer dieselbe Nuance des Braun zeigen, dass also einige dunkelbraun, andere wieder heller, noch andere fast gar nicht gefärbt erscheinen. Demnach muss die Substanz, welche diese eigenartige Farbenreaction mit der Chrom-

1) a. a. O. S. 624 .

9) H. Stilling, Du ganglion intercarotidien. Lausanne 1892. 
säure giebt, in den Zellen in ungleicher Quantität vorhanden sein. Ja häufig genug konnte ich feststellen, dass innerhalb der Zellenreihen vereinzelte Elemente zu bemerken waren, welche vollständig farblos geblieben waren. Dieselben hatten einen gut färbbaren Kern, helles ungefärbtes Protoplasma, welches mit stark lichtbrechenden, feinen, aber nicht gebräunten Körnchen durchsetzt war. Dennoch fühle ich mich nicht berechtigt zu der Annahme, dass die genannten Zellen verschiedene Arten darstellen, vielmehr erscheinen sie von einander nur verschieden in ihrem Gehalt an jener durch Chromsäure färbbaren Substanz. Es liegt nun sehr nahe diese rein quantitativen Differenzen von zeitlich verschiedenen physiologischen Zuständen der Elemente abhängig zu machen, eine Annahme, deren Wahrscheinlichkeit sich durch die nachfolgenden Befunde ergeben mag.

Mein Hauptinteresse nahmen aber stets die Venen, bezw. die venösen Räume in Anspruch, welche ja nach Arnold ${ }^{1}$ ) in den venösen Kreislauf eingeschaltet sind; und zwar will ich hier ein für alle Mal bemerken, dass zwischen den Befunden an den Venen und zwischen denen an den Venensinus kein principieller Unterschied zu bemerken war, dass also das, was ich von den Venen berichten werde, auch für die venösen Räume gilt.

Die Beobachtungen, die ich hier gleich beim ersten Blick in's Mikroskop machte, waren so merkwürdig, dass ich bei der ersten Nebenniere, die ich untersuchte - sie rührte von einem Rinde her - vermuthete, ich hätte es mit Artefacten oder irgend welchen pathologischen Erzeugnissen zu thun: Ich sah nehmlich in sämmtlichen Venen, seltener auch in den Arterien der Marksubstanz eine braune glasige, homogene Masse, welche innig mit Blutkörperchen vermengt war und meist einen Cylinder, also eine pralle Füllung des betreffenden Gefässes darstellte. Häufig auch zeigten sich, aber nur in den venösen Gefässen, Kugeln von derselben Beschaffenheit. Die Kugeln hatten meist einen dunkleren Farbenton angenommen, die sonst noch in den Venen vorhandenen glänzenden Massen zeigten gewöhnlich ein helleres Braun. Ausser diesen graduellen Differenzen, die übri-

1) a. a. 0. S. 94 . 
gens nicht immer deutlich waren, war jedoch kein Unterschied zwischen den Kugeln und den übrigen braunen Massen zu bemerken. Es handelte sich also augenscheinlich um Dinge derselben Art; Uebergänge von einem zum andern waren häufig zu constatiren. Diese braunen hyalinen Massen konnte ich dann bei jedem der genannten Thiere in der Marksubstanz feststellen. Niemals war an denselben auch nur eine Spur in denjenigen Nebennieren zu bemerken, welche nicht in Chromsäure, sondern in Alkohol fixirt worden waren.

Auch die Marksubstanzzellen bleiben bei directer Alkoholbehandlung farblos, und zwar ist nach den Angaben v. Brunn's ${ }^{x}$ ) schon eine 10-15 Minuten lange Einwirkung von Alkohol genügend, um die Chromsäurereaction zu vereiteln. Ich war deshalb schon jetzt versucht anzunehmen, dass jene braunen Massen in den Venen zu dem Inhalt der Marksubstanzzellen in einer nahen Beziehung ständen, vielleicht sogar mit denselben identisch. wären. -

Diese Befunde in den Venen sind so in die Augen springend, dass dieselben unmöglich übersehen werden können. Jedoch finden sich die braunen Massen deutlich nur in den Nebennieren, welche gleich nach dem Tode des betreffenden Thieres in die Chromsäurelösung gelegt sind. Auch dürfen die Organe beim Herausnehmen aus dem Cadaver nicht angeschnitten werden, weil man sonst meist nur leere Venen antrifft. Wahrscheinlich sind also bei den bisherigen Untersuchungen diese beiden Vorbedingungen nicht erfüllt werden.

Häufig waren diese braunen Massen untermischt mit Nebennierenzellen, Resten von solchen, oder auch nur isolirten Kernen.

Es galt nun diese braunen Massen bis in die Venenwurzeln und eventuell noch weiter zu verfolgen, um ihre Herkunft festzustellen. Auch die Wandung der venösen Gefässe bot Verhältuisse dar, welche wegen des raschen Einlegens der Organe in fixirende Flüssigkeiten deutlicher zu beobachten waren als bei den menschlichen Nebennieren. Manchmal waren nehmlich Zapfen von Nebennierensubstanz, ähnlich wie ich sie oben be- 
schrieben habe, schon in ganz grossen Venen zu bemerken. Besonders deutlich war dies Verhältniss bei den Nebennieren des Schlafes zu beobachten: hier zeigte sich, dass in die die Marksubstanz ihrer ganzen Länge nach durchziehende Centralvene eine Menge ziemlich langer Fortsätze hineinragten, welche aus Nebennierenzellen bestanden. Diese Fortsätze zeigten oft zu mehreren einen gemeinschaftlichen Ursprung in der die Vene begrenzenden Marksubstanz, so dass sie sich büschelförmig in das Lumen hinein erstreckten. Ein Endothel oder eine Wandung war weder auf der Höhe dieser Fortsätze noch an ihrer Ursprungsstelle za bemerken: die Zapfen drangen direct aus dem Parenchym in das Venenlumen hinein (s. Fig. 1).

Bei den kleineren Venen würden diese Fortsätze häufiger, setzten sich gleichfalls aus den braunen Marksubstanzzellen zusammen und schossen hier entweder aus dem Parenchym der Marksubstanz direct in das Venenlumen hinein, oder sassen der inneren Seite der Gefässwand mit einer mehr oder weniger breiten Basis auf; der Veneninhalt bestand auch hier aus Blutkörperchen, vereinzelten Nebennierenzellen und vor Allem aus jenen so auffallenden braunen glasigen Massen. Doch zeigte sich hier, und bei kleineren Venen wurde das noch deutlicher, ein ganz eigenthümliches Verhältniss zwischen diesen braunen, hyalinen Massen und dem Zellinhalt jener NebennierenzellenFortsätze: Die membranlosen Zellen dieser Zapfen communicirten deutlich mit jenen homogenen Massen; die letzteren lagen zum Theil noch in den Zellen, zum Theil schon im Venenlumen, so dass Zellinhalt und Veneninhalt ein zusammenhängendes Ganzes bildeten.

In den kleinsten Venen war noch eine engere Beziehung zwischen Marksubstanzzellen und dem braunen Veneninhalt zu bemerken: Hier zeigte das meist spaltförmige Gefässchen nur auf der einen Seite ein Endothel, auf der anderen waren jene braunen Marksubstanzzellen in einer Reihe aufgestellt, deren Inhalt wiederum mit dem Veneninhalt in der angegebenen Weise communicirte (s. Fig. 4 und 5). Kein Zweifel, dass sich hier ein directes Ueberfliessen jener braunengasen von Seiten der Marksubstanzzellen in den venösen Kreislauf feststellen liess. Bei dem engen Connex, in dem hier 
Veneninhalt und Zellinhalt standen, war es nicht weiter wunderbar, wenn sich hin und wieder einmal Blutkörperchen dem Zellprotoplasma beimischten; vielleicht ist so das verhältnissmässig häufige Auftreten von rothen Blutkörperchen in den Marksubstanzzellen zu erklären. Aus demselben Grunde wird auch der weitere schon oben bemerkte Befund, dass mehrfach Nebennierenzellen oder Bruchstücke derselben in den Venen angetroffen wurden, leicht verständlich.

Verfolgen wir jene kleinsten Gefässe noch weiter, so kommen wir schliesslich auf Gebilde, welche den Anschein erwecken, als ob diese braunen Massen ein Secret darstellen, welches direct von jenen braunen Zellen ausgeschieden wäre. Es waren dies Kanäle, welche eine sehr auffallende Aehnlichkeit mit Drüsenschläuchen hatten: Die bindegewebigen Septen der Marksubstanz bilden nehmlich, wie schon oben bemerkt, Schläuche, deren Wand auf der Innenseite mit eben jenen braunen Zellen besetzt ist; es kam nun darauf an in diesen scheinbar soliden, aus Marksubstanzzellen bestehenden Cylindern Lumina zu entdecken. Das ist mir nun in der That bei mehreren Rinds- und PferdeNebennieren gelungen: Zuerst zwar nur selten, später aber häufig genug, konnte ich feststellen, dass jene Zellcylinder in ihrer Mitte eine Lichtung zeigten, welche gewöhnlich mit denselben homogenen Massen ausgefüllt war, wie das ganze Venensystem der Marksubstanz. Auch hier zeigte sich denn, dass diese braunen Massen zum Theil noch in den Zellen des Cylinders lagen, so dass es den Anschein hatte, als ob sie eben im Begriffe wären, aus denselben heraus zu treten. Das Verhältniss dieser kleinen Kanäle wird durch die Figuren noch klarer werden (s. Fig. 6, 7, 8). Sowohl Längs- als Querschnitte jener Cylinder erscheinen angefüllt mit braunen Massen, welche zum Theil noch in den braunen Zellen liegen. Wie aus den Querschnittsfiguren hervorgeht, haben diese Kanäle oft ein ganz beträchtliches Lumen und unterscheiden sich von den vorher beschriebenen kleinsten Gefässchen nur dadurch, dass sie gar kein Endothel haben, vielmehr nur ein von membranlosen Nebennierenzellen gebildetes $\mathrm{R}_{0} \mathrm{hr}$ darstellen.

Auch diese vollständig endothellosen Kanälchen zeigen ausser ihrem braunen Inhalt nicht so selten ganz vereinzelte rothe Blut- 
körperchen. Manchmal gelang es mir auch, eine directe Communication eines solchen Kanälchens mit einem Capillargefäss deutlich zu constatiren.

An dieser Stelle möchte ich eines sehr eigenthümlichen, freilich vereinzelten Befundes gedenken: Bei einer Rindsnebenniere fielen in der Marksubstanz sogleich einige grosse, quer getroffene, runde, weit klaffende Lumina auf, welche einem ganz merkw ürdigem Kanalsystem entspracheu. Die Wand dieser Lumina bestand aus einer bindegewebigen Membran, auf welcher nach innen $z u$ eine ein- oder mehrfache Schicht von Marksubstanzzellen aufsass, so dass sich das Ganze also ausnahm, wie ein richtiger, weiter mit hohem Epithel ausgekleideter Drüsengang. Dieser Drüsengang war zum Theil ausgefüllt mit rothen Blutkörperchen, zum anderen Theil mit jenen hyalinen Kugeln und Massen, wie sie oben beschrieben sind. Auch hier liess sich, wie aus der Abbildung (s. Fig. 9) hervorgeht, ein directes Austreten dieser Massen aus den wandständigen Zellen nachweisen; und zwar war auch hier deutlich zu bemerken, dass die Kugeln eine tiefere Braunfärbung zeigten, als die sonst noch den Kanal ausfüllenden bräunlichen Massen. Das ganze Kanalsystem war so regelmässig durch die Nebenniere zu verfolgen, dass es nicht anging, einen pathologischen Vorgang, etwa multiple Hämorrhagien, als Veranlassung dieser eigenartigen Befunde anzusprechen. Entschieden lag vielmehr eine Combination von Drüsenkanälen und Gefässen vor. Denn die Kanäle, welche deutlichst mit Drüsenzellen ausgekleidet waren, führten sowohl Blut als jene so merkwürdigen braunen hyalinen Massen.

Ich habe mich bemüht, an anderen Rindsnebennieren diese Dinge wieder zu finden, aber leider keinen Erfolg gehabt: Der Befund an jener einen Nebenniere blieb vereinzelt. - Ausser jenen Kanälen zeigte diese Nebenniere an den übrigen Venen sowohl bezüglich des Veneninhaltes als der Wandungen dieselben Verhältnisse, wie sie oben bei allen anderen notirt sind.

Was nun die Eigenschaften dieser braunen Massen anbetrifft, so liess sich hier nicht viel Charakteristisches feststellen: Sie wurde nicht angegriffen von Schwefel-, Salpeter- und Essigsäure, desgleichen nicht von schwefelsaurer Magnesia, phosphorsaurer Magnesia, phosphorsaurem Ammoniak. Ich machte auch mehr- 
fach den Versuch durch 2-10procentige Höllenstein-Lösungen eine Veränderung an jenen homogenen Massen hervorzurufen, das ist mir jedoch niemals gelungen, selbst wenn ich das Präparat stark ansäuerte und erwärmte. Alle diese Reactionen wurden auch an Präparaten versucht, welche direct aus der Chromsäure kamen und nicht in Alkohol nachgehärtet waren, aber mit demselben Misserfolg: die braunen Massen wurden absolut nicht verändert.

Auch eine typische Färbung für diese Substanz vermag ich nicht anzugeben: Von Eosin wurde sie rosaroth gefärbt, sonst nahm dieselbe nur einen schwachen Schimmer der betreffenden Farbstoffe an, welcher die braune Farbe nur wenig verdeckte. Hier ist jedoch eine Ausnahme zu verzeichnen: Ausser anderen Färbungen versuchte ich auch die Russel'sche ${ }^{1}$ ) Methode: Sie besteht in einer successiven Behandlung der Schnitte mit Carbolfuchsin ( $2 \mathrm{pCt}$. gesättigte Lösung) 10 Minuten und Carboljodgrün (1:100 g 2 procentiger Carbollösung) 5 Minuten. Russel hat dieselbe angegeben zur Darstellung der sogenannten Russel'schen Fuchsinkörperchen, kleiner hyaliner Kügelchen in- und ausserhalb von Zellen, welche bei der genannten Methode eine tief rothe Farbe annehmen, während die Kerne grün erscheinen. Es ergab sich bei den Nebennieren nun das überraschende Resultat, dass ausser den Kernen auch die braunen hyalinen Massen ein intensiv grünes Colorit annahmen, während die rothen Blutkörperchen sich constant roth gefärbt zeigten. Durch das Nebeneinander beider Farben in einem Gefässe erhielt ich oft recht bunte Bilder. Versuche, diesen Veneninhalt in 4procentiger Sublimatoder in 10procentiger Salpetersäurelösung zu fixiren, misslangen vollständig.

Auffallend war es, dass von jenen Massen nichts in den Organen za bemerken war, welche nicht in Chromsäure, sondern direct in Alkohol fixirt worden waren. Die Substanz, welche dieses eigenartige glasige Produkt mit der Chromsäure giebt, muss also in Alkohol löslich sein.

Der Alkohol, in welchem die frischen Nebennieren gelegen haben, nimmt bald eine bräunliche bis tiefrothe Farbe an, auf

1) Lancet. 1890. 13. Dec. 
welche Thatsache Arnold ${ }^{1}$ ) schon vor vielen Jahren aufmerksam gemacht hat. Dass diese Färbung durch jene Substanz bedingt ist, kann ich natürlich nur vermuthen. Ich konnte nun constatiren, dass auch diejenigen Nebennieren, welche in Sublimat oder Salpetersäure fixirt worden sind, beim Einlegen in Alkohol dem letzteren eine rothe Farbe verleihen.

Ich will nun noch über einige Versuche berichten, welche ich machte, um die geschilderte Substanz aus der Vene aufzufangen und direct, also ohne vorherige Chromsäureeinwirkung unter das Mikroskop zu bringen.

$\mathrm{Zu}$ diesem $\mathrm{Zwecke}$ wurde einem ätherisirten Hunde die linke Vena suprarenalis unterbunden, kurze Zeit gewartet, dann die prall gefüllte Vene aufgeschnitten und das aufgefangene Blut auf den Objectträger gebracht. An 2 anderen lebenden Hunden wurde das Blut gleichfalls aus der Vena suprarenalis mit dem Objectträger entnommen, ohne vorherige Unterbindung der Vene.

Jedesmal, und es wurden viele Präparate gemacht, bemerkte ich in dem Blute eine grosse Menge kleiner, hyaliner, glänzender, vollständig farbloser Kügelchen, von der Grösse eines halben rothen Blutkörperchens, welche meist in Haufen bei einander lagen und weder zu den rothen, noch zu den farblosen Elementen des Blutes. in irgend einer Beziehung zu stehen schienen. Bei Osmiumsäurebehandlung wurden diese Kägelchen absolut nicht verändert. Aehnliche helle Gebilde hat Gottschau ${ }^{2}$ ) beim Kaninchen im Nebenuierenblut gesehen, welches er durch leichten Druck auf das Organ aus der Vene entleerte.

Es erübrigt jetzt noch möglichst objectiv zu prüfen, wie diese gesammten anatomischen Befunde physiologisch zu verwerthen sind.

Da handelt es sich denn, wie aus den oben berichteten Thatsachen hervorgeht, um die Frage: sind wir berechtigt auf Grund dieser Thatsachen anzunehmen, dass jene braunen hyalinen Massen ein Secret darstellen, welches von den braunen

2) a. a. 0.5 .575 . 
Zellen der Marksubstanz ausgeschieden und durch die Venen dem Kreislauf zugeführt sind? Diese Frage ist nicht so leicht zu beantworten. -

Erörtern wir zunächst das, was uns dazu führen könnte, diese Frage zu bejahen: Wir konnten feststellen, dass die braunen Zellen der Marksubstanz Schläuche bilden, welche, von einer bindegewebigen Hülle umgeben, in ihrem Inneren mit jenen braunen hyalinen Massen ausgefüllt sind. Eine Communication dieser Zellschläuche mit den Capillargefässen liess sich nachweisen. Es liess sich ferner constatiren, dass diese braunen Massen im engsten Zusammenhang mit dem Zellinhalt standen, so dass man in der That den Eindruck gewann, als ob jene eben im Begriff wären, aus den Zellen herauszutreten. Hiermit liesse sich auch die Thatsache in Einklang bringen, dass die Zellen eine verschiedene Intensität in der Braunfärbung zeigten; man könnte also annehmen, die einen hätten ihren Inhalt zum Theil oder ganz in jene Kanälchen ergossen, die anderen nicht.

Mit dem braunen Hyalin finden wir nun sämmtliche Venen der Marksubstanz ausgestopft, und zwar lassen hier die Wandungen Verhältnisse erkennen, welche uns gleichfalls zu der Ansicht verleiten können, dass das braune Hyalin von den braunen Zellen ausgeschieden wird und zwar hier direct in die Venen hinein. Wir sahen nehmlich, dass die kleinsten Venen auf ibren Quer- und Längsschnitten (s. Fig. 3. 4, 5) nur auf einer Seite eine endotheliale Wand besassen, dass sie auf der anderen von einer vollständig endothellosen Lage jener braunen Zellen begrenzt wurden, deren Inhalt wiederum mit dem braunen Veneninhalt in der angegebenen Weise zu communiciren schien. Bei den grösseren Venen kommt diese Neigung der Marksubstanzzellen, mit dem Veneninhalt in Communication zu treten, in jenen mehrfach beschriebenen Zapfen zum Ausdruck, welche, nur aus Marksubstanzzellen bestehend, meist ohne Endothelbekleidung frei in das Venenlumen hineinragten.

Aber ganz die gleichen braunen Massen - und das führt mich zu den Gründen, welche uns veranlassen können, die oben aufgeworfene Frage zu verneinen - finden sich auch in den Arterien. Es liess sich, wenn auch vereinzelt, feststellen, dass jene braunen Massen das Lumen der Arterien vollständig aus- 
füllten. - Man könnte hier also einfach sagen, das braune Hyalin ist in der Agone, oder post mortem, vielleicht gerade durch die Einwirkung der Chromsäure auf die noch lebenswarmen Zellen aus den letzteren in sämmtliche Gefässe ausgetreten, wie es $j$ a auch von manchem unter pathologischen Verhältnissen beobachteten Hyalin mehr als wahrscheinlich ist, dass es erst in der Agone oder vielleicht sogar post mortem aus den Zellen austritt. Nun bin ich allerdings nicht der Ansicht, dass das gewöhnliche pathologische Hyalin unserem braunen Hyalin gleich $\mathrm{zu}$ setzen ist; vielmehr unterscheidet sich letzteres von dem ersteren nicht nur durch die Chromsäurereaction, sondern vor Allem durch seine Löslichkeit in Alkohol.

Diese Thatsache, dass sich das braune Hyalin auch in den Arterien findet, könnte man nun mit dem Einwand beseitigen wollen, dass der Veneninhalt durch mannichfache Manipulationen beim Herausnehmen der Organe rückläufig in die Arterien hineingetrieben sein würde. Ich habe mich nun der grössten Vorsicht beim Einlegen der Nebennieren in die fixirenden Flüssigkeiten befleissigt und jedwede Einflüsse auf den Gefässinhalt, wie Druck mit der Hand u. s. w., zu vermeiden versucht, habe aber selbst dann noch in vereinzelten Arterien braune Massen gefunden. Auch das will indess nicht viel sagen, denn beim Schlachten und Ausweiden des Thieres passiren so vielerlei Lageveränderungen an den Organen, dass die Möglichkeit eines Zurückfliessens des Veneninhaltes in die Arterien gewiss niemals auszuschliessen ist.

Die Frage, ob das Auftreten dieses braunen Hyalins in den Gefässen einem intravitalen Vorgange entspricht, ist demnach als eine offene zu betrachten.

Wir sind also vorläufig noch nicht in der Lage, beweisen zu können, dass die beschriebenen braunen Massen ein Secret darstellen, welches von den Marksubstanzzellen ausgeschieden und durcb die Venen dem kreisenden Blute zugeführt wird.

Die gesammten, oben berichteten Befunde lassen jedoch auf eine sehr nahe Beziehung der Zellen der Nebennieren zu den Venen und der venösen Blatbahn schliessen. 


\section{Erklärung der Abbildungen.}

Tafel IV.

Vergrösserung: Zeiss DD Oc. 4.

Fig. 1. Aus der Wand der Centralvene einer Schafsnebenniere. Grosser und kleiner Marksubstanzzellenzapfen mit Blut und brauner homogener Masse im Lumen.

Fig. 2. Braunes Hyalin und Blut in grosser Vene der Marksubstanz (Rind).

Fig. 3. Kleine Vene der Marksubstanz mit Blutkörperchen, braunen homogenen Kugeln und Massen angefüllt. Auf der einen Seite, bei a, hat die Wand kein Endothel, sondern besteht nur aus Nebennierenzellen, auf der anderen mit Endotbel bekleideten Seite münden 2 kleine Aeste ein (Rind).

Fig. 4. Längsschnitt einer kleinen Vene mit Blut und homogenen braunen Massen; auf der einen Seite endotheliale Wand, auf der anderen besteht dieselbe aus mehr oder weniger braunen. Marksubstanzzellen (Pferd).

Fig. 5. Querschnitt einer kleinen Vene, wit braunem Hyalin gefüllt, weiche gleichfalls nur auf einer Seite Endothel zeigt (Pferd).

Fig. 6, 7, 8. Quer- und Längsschnitte von Markzellenkanälchen, mit braunem Hyalin gefültt (Rind).

Fig. 9. Vereinzelter Befund (Rind) s. S. 271. 